\title{
Erratum to: A proposal for the use of uniform diagnostic criteria for gestational diabetes in Europe: an opinion paper by the European Board \& College of Obstetrics and Gynaecology (EBCOG)
}

\author{
Katrien Benhalima $^{1}$ - Chantal Mathieu ${ }^{1}$ Peter Damm ${ }^{2}$ - André Van Assche ${ }^{3}$. \\ Roland Devlieger $^{3} \cdot$ Gernot Desoye $^{4} \cdot$ Rosa Corcoy $^{5,6} \cdot$ Tahir Mahmood $^{7} \cdot$ Jacky Nizard $^{8} \cdot$ \\ Charles Savona-Ventura ${ }^{9} \cdot$ Fidelma Dunne $^{10,11}$
}

Published online: 18 May 2016

(C) Springer-Verlag Berlin Heidelberg 2016

\section{Erratum to: Diabetologia \\ DOI 10.1007/s00125-015-3615-7}

Unfortunately, one of the affiliations for Rosa Corcoy was missing from this paper. The full details of the affiliations are listed here.

The online version of the original article can be found at http://dx.doi.org/ 10.1007/s00125-015-3615-7.

Katrien Benhalima

katrien.benhalima@uzleuven.be

1 Department of Endocrinology, UZ Gasthuisberg, KU Leuven, Herestraat 49, 3000 Leuven, Belgium

2 Center for PregnantWomen with Diabetes, Department of Obstetrics, Juliane Marie Centre, Rigshospitalet, Clinical Institute of Medicine, Faculty of Health and Medicine Sciences, University of Copenhagen, Copenhagen, Denmark

3 Department of Obstetrics \& Gynecology, UZ Gasthuisberg, KU Leuven, Leuven, Belgium

4 Department of Obstetrics \& Gynecology, Medical University of Graz, Graz, Austria

5 Endocrinology and Nutrition Department, Hospital de la Santa Creu I, Sant Pau, Barcelona, Spain
6 Centro de Investigación Biomédica en Red de Bioingeniería, Biomateriales y Nanomedicina (CIBER-BBN), Spain http://www.ciber-bbn.es

7 Department of Obstetrics \& Gynecology, University of Edinburgh, Edinburgh, UK

8 Department of Obstetrics \& Gynecology, AP-HP Groupe Hospitalier Pitié-Salpêtrière, Paris, France

9 Department of Obstetrics \& Gynecology, University of Malta Medical School, Msida, Malta

10 Endocrinology School of Medicine, National University of Ireland, Galway, Ireland

11 Galway Diabetes Research Centre, National University of Ireland, Galway, Ireland 